\title{
Order Properties of Conditional Warm Standby System at Random Time
}

\author{
Yu-Xia JIANG \\ School of Mathematics and Physics \\ Lanzhou Jiao tong University \\ Lanzhou, China \\ E-mail:1078479843@qq.com
}

\author{
Zheng-Cheng ZHANG \\ School of Mathematics and Physics \\ Lanzhou Jiao tong University \\ Lanzhou, China \\ E-mail:zhzhcheng004@163.com
}

\begin{abstract}
This article discusses the residual life and inactivity time of warm standby system at random time. The reliability function of the residual life(inactivity time) of the warm standby system at random time is obtained. And by using stochastic order, we compared the stochastic properties of residual life at different times.
\end{abstract} life

Keywords-Stochastic order; warm standby system; Residual

\section{INTRODUCTION}

In general, three types of standby redundancy are usually discussed in literature, namely, cold standby, hot standby, and warm standby. In a cold standby redundancy, the redundant component is inactive, that is, it has zero failure rate and hence never fails in standby state; in a system with hot standby redundancy, and the redundant component also works in the usual environment and may fail when it is in standby state. That is, the active component and the redundant one form a parallel system. The warm standby means that the components work in a warm environment when they are in standby status. Both the residual life and inactivity time of systems have been investigated in literature. For example, Asadi[1], Khaledi[2], Hashemi[3] , Zhang[4] .

Let $X$ be the lifetime of the redundancy in the usual environment with distribution function $F(t)$ and survival function $\bar{F}(t)=1-F(t)$. Since the redundant component in standby statu operates in a milder environment, the lifetime in standby statu should be stochastically larger than that in the usual environment. Light of the accelerated life model (see Bagdonavicius and Nikulin [5]) and the decelerated life model (see Singpurwalla [6]), the lifetime of the redundancy in standby status, say $X^{*}, F^{*}(t)$ be the distribution function of $X^{*}$. Since the standby unit is operated under milder environment than the usual level of environment during warm standby state, it is assumed that

$$
X^{*} \leq_{s t} X
$$

For any $t \geq 0,(1)$ implies that

$$
F^{*}(t)=F(\gamma(t))
$$

Where continuous non-decreasing function $\gamma(t)$ depends on the accelerated environment, $t \geq \gamma(t) \geq \gamma(0) \equiv 0$ for all $t \geq 0$.

According to the virtual age model (see Kijima[7],Finkelstein[8]), it is reasonable to assume that a standby redundancy switching to the usual environment at its age $t>0$ has the virtual age $\omega(t)$ satisfying (i) $t \geq \omega(t) \geq \omega(0) \equiv 0$ for all $t \geq 0 \quad$, (ii) $\omega(t)$ is continuous and increases in $t \geq 0$.

Let $Y$ be the lifetime of the active component with distribution function $G(t)$. Assume that $X$ and $Y$ are independent. According to $\mathrm{Li}$ et al [9], the total lifetime of the general standby system composed of the active component $Y$ and the standby one $X$ is

$$
T_{Y \mid X}= \begin{cases}Y, & \text { if } Y \geq X^{*} \\ Y+X_{\omega(Y)}, & \text { if } Y<X^{*}\end{cases}
$$

Here $\omega(t)=h(t, \gamma(t))$ for some bivariate function $h_{1}$, and

$$
X_{\omega(Y)}=X-\omega(Y) \mid X>\omega(Y)
$$

Is the residual life of the lifetime $X$ at random time $\omega(Y)$. The reliability function of the lifetime $T_{Y \mid X}$ of the standby system as below. For any $t \geq 0$,

$$
\bar{H}(t)=\bar{G}(t)+\int_{0}^{t} \frac{\bar{F}(t-\delta(u))}{\bar{F}(\omega(u))} \bar{F}(\gamma(u)) d G(u)
$$

Where $\delta(u) \equiv u-\omega(u)$ for all $u \geq 0$.

Definition 1[10] the lifetime of the component is assumed to be $M$, the residual life and inactivity time of the component at $t \geq 0$ are

$$
M_{t}=(M-t \mid M>t), M_{(t)}=(t-M \mid M \leq t) .
$$

The survival function is 


$$
\bar{H}_{t}(x)=\bar{H}(t+x) / \bar{H}(t), \bar{H}_{(t)}(x)=H(t-x) / H(t) .
$$

Definition 2[11] suppose $X$ and $Y$ have their distribution functions $F$ and $G$, denote $\bar{F}=1-F$ and $\bar{G}=1-G$ as their survival functions, respectively. For any $x \geq 0$

a) If $\bar{F}(x) \leq \bar{G}(x)$, then $X$ is said to be smaller than $Y$ in the usual stochastic order (denoted by $X \leq_{s} Y$ );

b) If $\bar{F}(x) / \bar{G}(x)$ is decreasing in $x \geq 0$, then $X$ is said to be smaller than $Y$ in the hazard rate order (denoted by $\left.X \leq_{h r} Y\right)$;

c) if $F(x) / G(x)$ is decreasing in $x \geq 0$, then $X$ is said to be smaller than $Y$ in the reversed hazard rate order (denoted by $X \leq Y$ ).

For ease of reference, relations among these orderings are presented as follows(see, e.g., Shaked M, Shanthikumar [12]):

$$
X \leq_{s t} Y \Rightarrow X \leq_{h r} Y, X \leq_{s t} Y \Rightarrow X \leq_{r h} Y
$$

This article discusses the residual life and inactivity time of the warm standby system, we consider the reliability function of the residual life (inactivity time) of the warm standby system at random time $\Theta$. The influence of time on the residual life of the system is studied.

\section{MAIN RESUlTS}

Think of $t$ as a nonnegative random variable, as $\Theta$. The distribution function and survival function of $\Theta$ are $H, \bar{H}$. In what follows, we consider the residual life (inactivity time) of the warm standby system at random time $\Theta \geq 0$. That is

$$
(T(X, Y)-\Theta \mid \min (X, Y) \geq \Theta) .
$$

Theorem 1. Let $R^{\Theta}(x)$ be the reliability function of the residual life $(T(X, Y)-\Theta \mid \min (X, Y) \geq \Theta)$. Then, for any $x \geq 0, \quad \theta \geq 0$,

$$
\begin{aligned}
R^{\Theta}(x)= & \int_{0}^{\infty} \int_{\theta}^{\infty} \frac{\bar{F}(x+\theta-u+\omega(u)) \bar{F}(\gamma(u))}{\bar{F}(\gamma(\theta)) \bar{F}(\omega(u)) \bar{G}(\theta)} \\
& d G(u) d H(\theta)+\int_{0}^{\infty} \frac{\bar{G}(\theta+x)}{\bar{G}(\theta)} d H(\theta) .
\end{aligned}
$$

Proof. For any $x \geq 0, \quad \theta \geq 0$, we have

$$
\begin{aligned}
& R^{\Theta}(x) \\
= & P(T(X, Y)-\Theta \geq x \mid \min (X, Y) \geq \Theta) \\
= & \frac{P(T(X, Y)-\Theta \geq x, \min (X, Y) \geq \Theta)}{P(\min (X, Y) \geq \Theta)} \\
= & \int_{0}^{\infty} \frac{P(T(X, Y)-\theta \geq x, \min (X, Y) \geq \theta)}{P(\min (X, Y) \geq \theta)} d H(\theta) .
\end{aligned}
$$

Among,

$$
P(\min (X, Y) \geq \Theta)=\bar{F}(\gamma(\theta)) \bar{G}(\theta),
$$

and,

$$
\begin{aligned}
& P(T(X, Y)-\theta>x, \min (X, Y) \geq \theta) \\
= & P(T-\theta>x, X>Y, Y \geq \theta) \\
& +P(T-\theta>x, X<Y, X \geq \theta) \\
= & P\left(Y+X^{*}>x+\theta, X>Y, Y \geq \theta\right) \\
& +P\left(Y>x+\theta, X^{*} \geq \theta\right) \\
= & \int_{0}^{\infty} P\left(Y+X^{*}>x+\theta, X>Y, Y \geq \theta \mid Y=u\right) d G(u) \\
& +\bar{G}(x+\theta) \bar{F}(\gamma(\theta)) \\
= & \int_{\theta}^{\infty}[P(X-\omega(u)>x+\theta-u \mid X>u, Y=u) \\
& P(X>u \mid Y=u)] d G(u)+\bar{G}(x+\theta) \bar{F}(\gamma(\theta)) \\
= & \int_{\theta}^{\infty} \frac{\bar{F}(x+\theta-u+\omega(u))}{\bar{F}(\omega(u))} \bar{F}(\gamma(u)) d G(u) \\
& +\bar{G}(x+\theta) \bar{F}(\gamma(\theta)) .
\end{aligned}
$$

Hence,

$$
\begin{aligned}
R^{\Theta}(x)= & \int_{0}^{\infty} \int_{\theta}^{\infty} \frac{\bar{F}(x+\theta-u+\omega(u)) \bar{F}(\gamma(u))}{\bar{F}(\gamma(\theta)) \bar{F}(\omega(u)) \bar{G}(\theta)} \\
& d G(u) d H(\theta)+\int_{0}^{\infty} \frac{\bar{G}(\theta+x)}{\bar{G}(\theta)} d H(\theta) .
\end{aligned}
$$

The following theorem is illustrated the influence of time on the residual life of the system is studied.

Theorem 2. If $\Theta_{1} \leq_{s t} \Theta_{2}$, then

$$
\begin{aligned}
& \left(T(X, Y)-\Theta_{1} \mid \min (X, Y) \geq \Theta_{1}\right) \\
& \geq_{s t}\left(T(X, Y)-\Theta_{2} \mid \min (X, Y) \geq \Theta_{2}\right) .
\end{aligned}
$$

Proof. Due to Theorem 1, for any $x \geq 0, \Theta \geq 0$, we 
have

$$
\begin{aligned}
R^{\Theta_{1,2}}(x)= & \int_{0}^{\infty} \int_{\theta_{1,2}}^{\infty} \frac{\bar{F}\left(x+\theta_{1,2}-u+\omega(u)\right) \bar{F}(\gamma(u))}{\bar{F}\left(\gamma\left(\theta_{1,2}\right)\right) \bar{F}(\omega(u)) \bar{G}\left(\theta_{1,2}\right)} \\
& d G(u) d H\left(\theta_{1,2}\right)+\int_{0}^{\infty} \frac{\bar{G}\left(\theta_{1,2}+x\right)}{\bar{G}\left(\theta_{1,2}\right)} d H\left(\theta_{1,2}\right),
\end{aligned}
$$

So,

$$
\begin{aligned}
& R^{\Theta_{1}}(x)-R^{\Theta_{2}}(x) \\
= & \int_{0}^{\infty} \int_{\theta_{1}}^{\infty} \frac{\bar{F}\left(x+\theta_{1}-u+\omega(u)\right) \bar{F}(\gamma(u))}{\bar{F}\left(\gamma\left(\theta_{1}\right)\right) \bar{F}(\omega(u)) \bar{G}\left(\theta_{1}\right)} d G(u) d H\left(\theta_{1}\right) \\
& +\int_{0}^{\infty} \frac{\bar{G}\left(\theta_{1}+x\right)}{\bar{G}\left(\theta_{1}\right)} d H\left(\theta_{1}\right)-\int_{0}^{\infty} \frac{\bar{G}\left(\theta_{2}+x\right)}{\bar{G}\left(\theta_{2}\right)} d H\left(\theta_{2}\right) \\
& -\int_{0}^{\infty} \int_{\theta_{2}}^{\infty} \frac{\bar{F}\left(x+\theta_{2}-u+\omega(u)\right) \bar{F}(\gamma(u))}{\bar{F}\left(\gamma\left(\theta_{2}\right)\right) \bar{F}(\omega(u)) \bar{G}\left(\theta_{2}\right)} d G(u) d H\left(\theta_{2}\right) \\
\geq & 0,
\end{aligned}
$$

Hence,

$$
\begin{aligned}
& \left(T(X, Y)-\Theta_{1} \mid \min (X, Y) \geq \Theta_{1}\right) \\
& \quad \geq_{s t}\left(T(X, Y)-\Theta_{2} \mid \min (X, Y) \geq \Theta_{2}\right) .
\end{aligned}
$$

By Theorem 2, we can easily obtain the following theorem.

Theorem 3. If $\Theta_{1} \leq_{h r} \Theta_{2}$, then

$$
\begin{gathered}
\left(T(X, Y)-\Theta_{1} \mid \min (X, Y) \geq \Theta_{1}\right) \geq_{s t} \\
\left(T(X, Y)-\Theta_{2} \mid \min (X, Y) \geq \Theta_{2}\right) .
\end{gathered}
$$

Theorem 4. If $\Theta_{1} \leq_{r h} \Theta_{2}$, then

$$
\begin{aligned}
& \left(T(X, Y)-\Theta_{1} \mid \min (X, Y) \geq \Theta_{1}\right) \\
& \quad \geq_{s t}\left(T(X, Y)-\Theta_{2} \mid \min (X, Y) \geq \Theta_{2}\right) .
\end{aligned}
$$

Theorem 3 and Theorem 4 show that in the case of hazard rate order and reversed hazard rate order, the same conclusion as Theorem 2 can be obtained, i.e., the residual life of the system decreases as time increases.

Then, we consider the inactivity time of the warm standby system at random time $\Theta \geq 0$. That is

$$
(\Theta-T(X, Y) \mid \max (X, Y) \leq \Theta)
$$

Theorem 5. Let $R^{(\Theta)}(x)$ be the reliability function of the inactivity time $(\Theta-T(X, Y) \mid \max (X, Y) \leq \Theta)$. Then, for any $x \geq 0, \quad \theta \geq 0$,

$$
\begin{aligned}
R^{(\Theta)}(x)= & \int_{0}^{\infty} \int_{0}^{\theta} \frac{F(\theta-x-u+\omega(u))}{F(\theta) G(\theta) \bar{F}(\omega(u))} \bar{F}(\gamma(u)) \\
& d G(u) d H(\theta)+\int_{0}^{\infty} \frac{F(\gamma(\theta)) G(\theta-x)}{F(\theta) G(\theta)} d H(\theta) .
\end{aligned}
$$

Proof. For any $x \geq 0, \quad \theta \geq 0$, we have

$$
\begin{aligned}
& R^{(\Theta)}(x) \\
= & P(\Theta-T(X, Y) \geq x \mid \max (X, Y) \leq \Theta) \\
= & \int_{0}^{\infty} \frac{P(\theta-T(X, Y) \geq x, \max (X, Y) \leq \theta)}{P(\max (X, Y) \leq \theta)} d H(\theta)
\end{aligned}
$$

Among,

$$
P(\max (X, Y) \leq \theta)=F(\theta) G(\theta),
$$

and,

$$
\begin{aligned}
& P(T(X, Y) \leq \theta-x, \max (X, Y) \leq \theta) \\
= & P(T(X, Y) \leq \theta-x, X<Y \leq \theta) \\
& +P(T(X, Y) \leq \theta-x, Y<X \leq \theta) \\
= & P\left(Y \leq \theta-x, X^{*} \leq \theta\right)+P\left(Y+X^{*} \leq \theta-x, Y<X \leq \theta\right) \\
= & G(\theta-x) F(\gamma(\theta)) \\
& +\int_{0}^{\infty} P\left(Y+X^{*} \leq \theta-x \mid X>u, Y=u\right) d G(u) \\
= & G(\theta-x) F(\gamma(\theta))+ \\
& \int_{0}^{\theta} P(X-\omega(u) \leq \theta-x-u \mid X>\omega(u)) \\
& P(X>u \mid Y=u) d G(u) \\
= & G(\theta-x) F(\gamma(\theta)) \\
& +\int_{0}^{\theta} \frac{F(\theta-x-u+\omega(u))}{\bar{F}(\omega(u))} \bar{F}(\gamma(u)) d G(u) .
\end{aligned}
$$

hence,

$$
\begin{aligned}
R^{(\Theta)}(x)= & \int_{0}^{\infty} \int_{0}^{\theta} \frac{F(\theta-x-u+\omega(u))}{F(\theta) G(\theta) \bar{F}(\omega(u))} \bar{F}(\gamma(u)) d G(u) d H(\theta) \\
& +\int_{0}^{\infty} \frac{F(\gamma(\theta)) G(\theta-x)}{F(\theta) G(\theta)} d H(\theta) .
\end{aligned}
$$

\section{CONCLUSIONS}

In this work, we investigate the reliability function of the residual life (inactivity time) of the warm standby system at random time $\Theta$. And compare the stochastic properties of Residual life at different times, the results show that with the increase of time, the residual life of the system becomes 
smaller and smaller.

\section{ACKNOWLEDGMENT}

The authors would like to thank both the referee and the editor for comments which helped to improve the presentation of this manuscript. This work is supported by National Natural Science Foundation of China(71361020).

\section{REFERENCES}

[1] Asadi M, Bairamov I.A note on the mean residual life function of a parallel system [J]. Communication in Statistics-Theory and Methods, 2005(34):475-484.

[2] Hashemi M, Tavangar M, Asadi M.Some properties of the residual lifetime of progressively Type- II right censored order statistics[J]. Statistics and Probability Letters, 2010(80):845-859.

[3] Khaledi B, Shaked M.Ordering conditional lifetimes of coherent systems [J]. Journal of Statistical Planning Inference, 2007(137):1173 -1184 .

[4] Zhang Z C, Yang Y.Ordered properties on the residual life and inactivity time of $(n-k+1)$-out-of-n systems under double monitoring [J]. Statistics and Probability Letters, 2010(80):711-717.

[5] Bagdonavicius V, Nikulin M. Accelerated Life Models: Modeling and Statistical Analysis [M]. Boca Raton,FL:Chapman \& Hall/CRC, 2001.

[6] Singpurwalla N D. Survival in Dynamic Environments [J]. Statistical Science, 1995, 10(1):86-103.

[7] Kijima M. Some Results for Repairable Systems with General Repair [J]. Journal of Applied Probability, 1987, 26(1):89-102.

[8] Finkelstein M S. On Statistical and Information-based Virtual Age of Degrading Systems [J]. Reliability Engineering and System Safety, 2007, 92(5):676 -681.

[9] Li X, Zhang Z, Wu Y. Some New Results Involving General Standby Systems [J]. Applied Stochastic Models in Busuiness and Industry, 2009, 25(5):632-642.

[10] Ross S M. Stochastic process and their applications [M]. New York: Wiley, 1996.

[11] Shaked M, Shanthikumar J G. Stochastic Orders [M]. New York: Springer, 2007.

[12] Shaked M, Shanthikumar J G. Stochastic orders and their applications [M].San Diego, CA: Academic Press, 1994. 